Volume 8, No.6, November - December 2019

International Journal of Advanced Trends in Computer Science and Engineering

Available Online at http://www.warse.org/IJATCSE/static/pdf/file/ijatcse147862019.pdf

https://doi.org/10.30534/ijatcse/2019/147862019

\title{
Novel Technique For PAPR and Average Power Reduction In OFDM Scheme
}

\author{
Adnan Haider Yusef Sa'd ${ }^{1}$, Aeizaal Azman Abd Wahab ${ }^{2}$ \\ ${ }^{1}$ School of Electrical \& Electronic Engineering, Universiti Sains Malaysia, Malaysia, \\ eng.adnanhaider@gmail.com \\ ${ }^{2}$ School of Electrical \& Electronic Engineering, Universiti Sains Malaysia, Malaysia, aeizaal@usm.my
}

\begin{abstract}
Orthogonal frequency division multiplexing (OFDM) is a multicarrier modulation technique that relies on orthogonality feature to increase transmission data rate while enhancing spectral efficiency at the same time. OFDM's major issue is the high peaks or peak-to-average power ration (PAPR) that introduced due to summation of subcarriers' signal. Many methods has been proposed to alleviate this issue however some may increase signal average power. In this paper, a new scrambling method using simple XOR operation is proposed for PAPR and average power reduction in OFDM system.
\end{abstract}

Key words : OFDM, PAPR, XOR, Power.

\section{INTRODUCTION}

Orthogonal Frequency Division Multiplexing (OFDM) is an amazing transmission technique that provide higher data rate along with implementation simplicity, and has an ability to cope with severe channel conditions. Moreover, unlike other techniques that utilize parallelism for increasing transmission speed, OFDM does use bandwidth efficiently by utilizing orthogonality feature. OFDM got adopted in many standards for its advantages over other techniques such as single carrier or frequency division multiplexing FDM. Such well-known standards are DSL, 802.11a, WiFi-Max, LTE, etc[1-3].

OFDM become more applicable and famous due to the advances in DSP technology however OFDM still suffer of one major problem that is the signal high peaks. The high peaks problem or what is so called the high peak-to-average ratio (PAPR) problem is a result of summation of multiple subcarriers' signal due to adopting parallelism concept in transmission. The high peaks of OFDM signal cannot be amplified linearly using normal power amplifier (PA) thus the signal is corrupted as high peaks lies in non-linear region of PA. So, as using normal PA with OFDM introduce in-band and out-band radiation OFDM require very complex PA design to maintain linearity. Mathematically, PAPR is calculated as follows:

$$
\operatorname{PAPR}(x[n])=\frac{\max \left(|x[n]|^{2}\right)}{E\left(|x[n]|^{2}\right)}
$$

where E denotes expectation.

There are many methods was proposed to alleviate the high PAPR issue, however, each one has advantages and disadvantages. An example of PAPR reduction techniques found in literature are PTS[4-6], SLM[4], TI[7], TR[8], interleaving[3], hybrid[9-13], etc.

The aforementioned techniques reduces PAPR of OFDM signal. However, if some maintain signal average power intact like in rotation based PAPR reduction techniques others increases the average power of the signal processed like in TI and TR. Coding, also, is used in PAPR reduction but it may increase signal average power as well.

In this paper a new approach is proposed to reduce average power along with PAPR of the OFDM signal by tweaking the selecting function. Moreover, symbol can have maximum of $M$ alternative symbol instead of 4 in rotation based techniques.

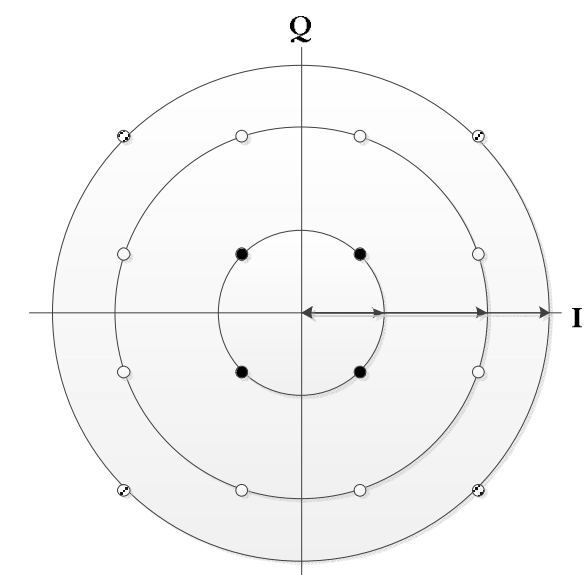

Figure 1: Constellation diagram: Rotation change signal phase but maintain amplitude. 


\section{SYMBOL ROTATION TECHNIQUE}

Symbol rotation technique is adopted in PTS and SLM. Rotation does not change signal's amplitude but the phase as it illustrated clearly in Fig. 1. The signal phase can be rotated from 0 to $2 \pi$ however for implementation simplicity only two or four rotation phases are used that's $[0, \pi]$ or $\left\{0, \pi, \pm \frac{\pi}{2}\right]$ which gives the following values as rotation factors $\{ \pm 1\}$ or $\{ \pm 1, \pm j]$. Therefore, as there are few rotation phases are used the constructed/selected rotation phase vectors play a very important role in affecting the performance of PAPR reduction of rotation based PAPR reduction techniques.

\section{PROPOSED METHOD}

In the proposed technique illustrated in Fig.2, the input data is scrambled for $U$ times using XOR operation with $U$ scrambling vectors before serial to parallel function so that:

$$
D^{(u)}=D \oplus B^{(u)} ; u=1,2, \ldots, U
$$

where $\oplus$ denotes XOR operation, $D$ is the input data and $B^{(u)}$ is the $u^{\text {th }}$ scrambling vector. If technique is implemented using processor with 64-bit width registers, scrambling can be done in one operation for 64 subcarriers configuration.

After scrambling the original input data, each scrambled data will be converted to parallel form using serial-to-parallel function and then modulated using symbol mapping modulation before applying the inverse fourier transform (IFFT). Finally, only one signal out of $U$ output of IFFT will be selected for transmission and the selected signal should have the lowest maximum peak or the lowest PAPR. The selected signal is given by

$$
s=\min \max \left(s^{(w)}\right)
$$

where minmax is the selection function.

At receiver side, based on the side information (SI) provided by the sender, the original data will be retrieved using XOR operation with same scrambling vector that used with the selected signal for transmit at the transmitter side. So, the original message is given by

$$
\bar{D}=D_{r} \oplus B^{\text {बas }}
$$

where $\oplus$ denotes XOR operation, $D_{\Gamma}$ represents received data binary sequence and $\hat{u}$ represents the index of selected scrambled data at the transmitter side for this transit.

The XOR based scrambling technique illustrated in Fig. 2 has the ability to produce an alternative signal with low PAPR. However, the resulted OFDM signal of such method might have a higher average power than the original signal.

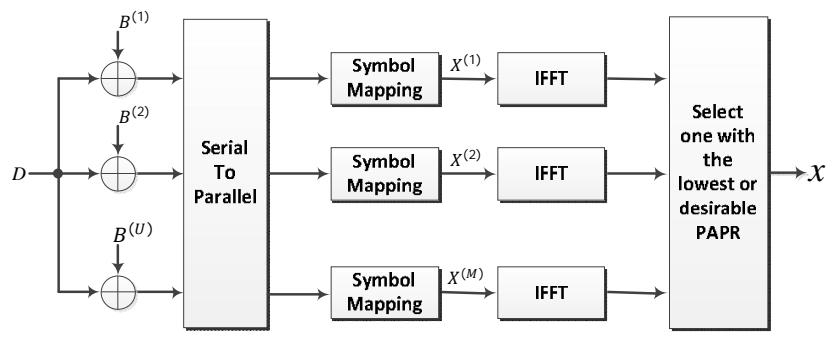

Figure 2: Block diagram of proposed technique. From constellation diagram perspective, unlike rotation based techniques, XOR based scrambling PAPR reduction technique can map any symbol from one constellation point into any one of $M$ other constellation points of $M$-ary symbol mapping modulation, instead of only four maximum achieved by rotation based techniques, using only simple XOR bitwise operation. For this reason, the selected signal with the lowest PAPR might have higher average power if only the used $M$-ary Modulation has more than one amplitude. Table 1 illustrates the impact of XOR on symbol's amplitude of 16-QAM modulation that have 3 amplitudes where 1 and 3 labels in table indicate lowest and maximum amplitude respectively. Colors in Table 1 indicate the impact on symbol amplitude where white denotes unchanged, green indicates reduction and red indicates increase.

\begin{tabular}{|c|c|c|c|c|c|c|c|c|c|c|c|c|c|c|c|c|}
\hline 5 & 0 & 1 & 2 & 3 & 4 & 5 & 6 & 7 & 8 & 9 & 10 & 11 & 12 & 13 & 14 & 15 \\
\hline 0 & 3 & 2 & 2 & 3 & 2 & 1 & 1 & 2 & 2 & 1 & 1 & 2 & 3 & 2 & $?$ & 3 \\
\hline 1 & 2 & 3 & 3 & 2 & 1 & 2 & 2 & 1 & 1 & 2 & 2 & 1 & 2 & 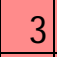 & 3 & 2 \\
\hline 2 & 2 & 3 & 3 & 2 & 1 & 2 & 2 & 1 & 1 & 2 & 2 & 1 & 2 & 3 & 3 & 2 \\
\hline 3 & 0 & 2 & 2 & 3 & 2 & 1 & 1 & 2 & 2 & 1 & 1 & 2 & 4 & 2 & 2 & 3 \\
\hline 4 & 2 & 1 & $\perp$ & 2 & 3 & 2 & 2 & 3 & 3 & 2 & 2 & 3 & 2 & 1 & 1 & 2 \\
\hline 5 & 1 & 2 & 2 & 1 & 2 & 3 & 3 & 2 & 2 & 3 & 3 & 2 & 1 & 2 & 2 & 1 \\
\hline 6 & 1 & 2 & 2 & 1 & 2 & 3 & 3 & 2 & 2 & 3 & 3 & 2 & 1 & $?$ & 2 & 1 \\
\hline 7 & 2 & 1 & 1 & 2 & 3 & 2 & 2 & 3 & 3 & 2 & 2 & 3 & 2 & 1 & 1 & 7 \\
\hline 8 & 2 & 1 & 1 & 2 & 3 & 2 & 2 & 3 & 3 & $\angle$ & 2 & 3 & 2 & 1 & 1 & 2 \\
\hline 9 & 1 & 2 & $\angle$ & 1 & 2 & 3 & 3 & 2 & 2 & 3 & 3 & 2 & 1 & 2 & 2 & 1 \\
\hline 10 & 1 & 2 & 2 & 1 & 2 & 3 & 3 & 2 & 2 & 3 & 3 & 2 & 1 & 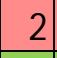 & 2 & 1 \\
\hline 11 & 2 & 1 & 1 & 2 & 3 & 2 & 2 & 3 & 3 & 2 & 2 & 3 & 2 & 1 & 1 & 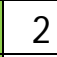 \\
\hline 12 & 3 & 2 & 2 & 3 & 2 & 1 & 1 & 2 & 2 & - & 1 & 2 & 3 & ? & 2 & 3 \\
\hline 13 & 2 & 3 & 3 & 2 & 1 & 2 & 2 & 1 & 1 & - & 2 & 1 & 2 & 2 & 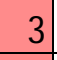 & 2 \\
\hline 14 & 2 & 3 & 3 & 2 & 1 & 2 & 2 & 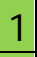 & 1 & 2 & 2 & 1 & 2 & 3 & 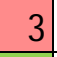 & 2 \\
\hline 15 & 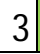 & 2 & & & 2 & & 1 & & 2 & & 1 & 2 & 3 & 2 & 2 & 3 \\
\hline
\end{tabular}

Table 1: XOR Impact on symbol amplitude of 16-QAM. D and B represent data and scrambler respectively

It can be seen clearly from Table. 1 that some values does not change the symbol amplitude however others do. Therefore, 
to maintain symbol power intact scrambler sequences has to be formed from the group of values that unchanged symbol's amplitude. In the other hand, to change signal's average power the scrambler sequence should be constructed using other values shown in Table. 1 that change symbol amplitude.

To achieve both PAPR reduction and maintain or even reducing the average power of the transmitted signal this paper propose tweaking the "minmax" function responsible for selecting the best result of multiple iterations to include acceptable average power condition in addition to the lowest PAPR feature condition. In other words, the lowest PAPR condition will only be examined if the resulted signal of iteration process of XOR scrambling based PAPR reduction has average power equal to or less than the average power of original signal. As a result, PAPR reduction is achieved while decreasing the average power of the transmitted signal.

\section{CONCLUSION}

In this paper XOR method was utilized as simple scrambling method for PAPR reduction in OFDM. Unlike rotation based techniques, the proposed method allows for generating $\mathrm{M}$ alternative symbol for a symbol modulated using M-ary modulation instead of maximum of 4 . Furthermore, the proposed approach reduces signal average power along with PAPR at the same time.

\section{ACKNOWLEDGEMENT}

This research was supported by Research University Grant, Universiti Sains Malaysia (1001/PELECT/814245).

\section{REFERENCES}

1. S. H. Han and J. H. Lee. An overview of peak-to-average power ratio reduction techniques for multicarrier transmission, IEEE Trans. Wireless Communications, vol. 12, pp. 56-65, 2005. https://doi.org/10.1109/MWC.2005.1421929

2. T. Jiang and $\mathrm{Y}$. Wu. An overview: peak-to-average power ratio reduction techniques for OFDM signals, IEEE Trans. Broadcasting, vol. 54, p. 257, 2008.

3. Y. Rahmatallah and S. Mohan. Peak-to-average power ratio reduction in OFDM systems: A survey and taxonomy, IEEE Trans. Communications Surveys \& Tutorials, vol. 15, pp. 1567-1592, 2013.

4. S. H. Müller, R. W. Bäuml, R. F. Fischer, and J. B. Huber. OFDM with reduced peak-to-average power ratio by multiple signal representation, in Annales des télécommunications, 1997, pp. 58-67.

5. S. H. Muller and J. B. Huber. A novel peak power reduction scheme for OFDM, in Personal, Indoor and Mobile Radio Communications, 1997. Waves of the Year
2000. PIMRC'97., The 8th IEEE International Symposium on, 1997, pp. 1090-1094.

6. S. H. Müller and J. B. Huber. OFDM with reduced peak-to-average power ratio by optimum combination of partial transmit sequences, Electronics letters, vol. 33, pp. 368-369, 1997. https://doi.org/10.1049/el:19970266

7. T. Wattanasuwakull and W. Benjapolakul. PAPR Reduction for OFDM Transmission by using a method of Tone Reservation and Tone Injection, in Information, Communications and Signal Processing, 2005 Fifth International Conf., 2005, pp. 273-277.

8. S.-E. Park, S. Yun, J. Y. Kim, D. S. Park, and P. Joo. Tone reservation method for PAPR reduction scheme, Project IEEE, vol. 802, 2003.

9. A. E. Jones, T. A. Wilkinson, and S. Barton. Block coding scheme for reduction of peak to mean envelope power ratio of multicarrier transmission schemes, Electronics letters, vol. 30, pp. 2098-2099, 1994. https://doi.org/10.1049/el:19941423

10. Z. Wang and S. Chen. Reduction PAPR of OFDM Signals by Combining Grouped DCT Precoding with PTS, Journal of Signal and Information Processing, vol. 5, p. 135, 2014. https://doi.org/10.4236/jsip.2014.54016

11. Sa'd, A.H.Y., A.A.A. Wahab, and H.H.Y. Sa'ad. A new precoded partial transit sequence method using the discrete cosine transform for peak-to-average power ratio reduction in an orthogonal frequency division multiplexing system, SCIENCEASIA, 2016. 42(6): p. 423-426.

12. Wahab, A. and A.H.Y. Sa'd. An improved IDCT-OFDM with partial transmit sequence, in 9th International Conference on Robotic, Vision, Signal Processing and Power Applications. 2017. Springer.

13. Liang, L. Y., Wahab, A. A. A., Alhady, S. S. N., \& Othman, W. A. F. W. Discrete wavelet transform with discrete cosine transform (DCT) for peak-to-average power ratio (PAPR) reduction in OFDM system, International Journal of Advanced Trends in Computer Science and Engineering, vol. 8, no. 5, pp. 2260-2264, 2019.

https://doi.org/10.30534/ijatcse/2019/62852019 